\title{
Peak-to-Average Power Ratio Reduction Based on Penalty-CCCP for Filter Bank Multicarrier Systems
}

\author{
Fangyu Cui, Yunlong Cai, Minjian Zhao, Ming Lei, and Lajos Hanzo
}

\begin{abstract}
Filter bank multicarrier (FBMC) has received substantial research attention as benefit of its low out-of-band emission and high spectral efficiency. However, a main challenge of FBMC is its relatively high peak-to-average power ratio (PAPR). Hence we formulate an optimization problem for the PAPR reduction of FBMC signals, where the PAPR is minimized subject to the constraints of symbol distortion and dummy subcarrier power used for PAPR-reduction. In order to tackle the highly coupled nonconvex problem, we convert the original problem into a more tractable yet equivalent form and then propose a novel algorithm based on the penalty concave-convex procedure (penalty-CCCP). Our simulation results show that the proposed algorithm substantially reduces the PAPR compared to conventional techniques.
\end{abstract}

Index Terms-PAPR reduction, FBMC, penalty concaveconvex procedure.

\section{INTRODUCTION}

In recent years, filter bank multicarrier (FBMC) has attracted much attention as a promising technique for nextgeneration systems [1], [2]. Due to the multicarrier feature, FBMC has a similar high peak-to-average power ratio (PAPR) issue as the traditional orthogonal frequency division multiplexing (OFDM) [3], [4], which degrades the efficiency of the power amplifier (PA). However, the conventional PAPR reduction methods conceived for OFDM [5], [6] suffer from significant performance degradation in FBMC systems due to the overlap between adjacent symbols. Hence, specific PAPR reduction methods have been designed for FBMC systems to handle the overlapping structure [7]-[12].

In [7] and [8], the partial transmit sequence (PTS) method is employed for the PAPR reduction of FBMC systems. Specifically, the phase factor vectors for multiple symbol blocks are jointly optimized in order to tackle the overlap between adjacent symbol blocks. In [9] and [10], the authors utilized the discrete Fourier transform (DFT) spreading to suppress the PAPR of FBMC, which is similar to the singlecarrier frequency-division multiple access (SC-FDMA). Since

This work was supported in part by the National Natural Science Foundation of China under Grant 61831004, in part by the Zhejiang Provincial Natural Science Foundation for Distinguished Young Scholars under Grant LR19F010002, and in part by the Fundamental Research Funds for the Central Universities under Grant 2018QNA5005. (Corresponding authors: Ming Lei; Yunlong Cai.)

F. Cui, Y. Cai, M. Zhao and M. Lei are with the College of Information Science and Electronic Engineering, Zhejiang University, Hangzhou 310027, China (e-mail: cfy531@zju.edu.cn; ylcai@zju.edu.cn; mjzhao@zju.edu.cn; lm1029@zju.edu.cn). L. Hanzo is with the Department of ECS, University of Southampton, U.K. (Email: lh@ecs.soton.ac.uk).

L. Hanzo would like to acknowledge the financial support of the Engineering and Physical Sciences Research Council projects EP/Noo4558/1, EP/PO34284/1, COALESCE, of the Royal Society's Global Challenges Research Fund Grant as well as of the European Research Council's Advanced Fellow Grant QuantCom. the transformations in the PTS and DFT spreading methods are known to the receiver and can be easily recovered, the bit error rate (BER) performance will not be affected. However, the PAPR reduction performance of these two types of methods is limited. In order to further reduce the PAPR of FBMC, researchers proposed the heuristic tone reservation (TR) [11] and iterative clipping and filtering (ICF) [12] methods. Due to the introduction of signal distortion, the PAPR of FBMC can be suppressed to a very low level at the cost of degraded BER performance. Note that appropriately designed signal distortion could efficiently reduce the PAPR with marginal BER performance degradation. Hence, the authors of [12] maximized the signal-to-distortion ratio in each iteration of the ICF method. However, the interior-point method is used to solve the formulated second-order cone programming (SOCP) problem, which results in a high complexity order.

In this work, we develop a novel PAPR reduction algorithm based on the penalty concave-convex procedure (penaltyCCCP) [13] for FBMC systems. The main contributions are summarized as follows:

1) We formulate the optimization problem of the PAPR reduction for FBMC systems. In particular, we minimize the PAPR of a frame of FBMC signals by optimizing the distorted frequency domain symbols subject to the constraint of the distortion on data subcarriers and the power constraint on the dummy subcarriers used for PAPR-reduction, which to the best of our knowledge has not been investigated before.

2) We convert the original challenging problem into a more tractable yet equivalent form and develop a penalty-CCCP based algorithm to solve it. Specifically, the subproblems in each iteration can be solved by using the Lagrange multiplier method or by simply examining the first-order optimality condition, whose complexity is much lower than the traditional interior-point method.

3) We analyze the computational complexity of the proposed algorithm and evaluate its performance through simulations. Our simulation results show that the proposed algorithm substantially reduces the PAPR of FBMC signals at a marginally reduced BER performance.

This paper is structured as follows. In Section II, we describe the system model and formulate the optimization problem of interest. In Section III, we develop the penaltyCCCP based algorithm and provide the complexity analysis. The simulation results are presented in Section IV and the conclusions are drawn in Section V. 


\section{System Model And Problem Formulation}

Consider an FBMC system with $N$ subcarriers, and assume that each frame consists $M$ symbol blocks. The oversampling rate is $L$ and the overlapping factor is denoted as $\beta$. Hence, the time-domain length of an FBMC frame is denoted as $K=$ $(M+\beta-1 / 2) L N$. Generally, the oversampling rate should satisfy $L \geq 4$ in order to calculate the PAPR of FBMC signals accurately. Dummy subcarriers are routinely used for PAPRreduction in multicarrier systems, hence mitigating the outof-band (OOB) emissions or for potential future applications instead of transmitting data [6]. We denote the set of data subcarriers as $\mathcal{N}$. Therefore, a frame of transmitted FBMC signal can be expressed as

$$
\begin{array}{r}
s(k)=\sum_{m=0}^{2 M-1} \sum_{n \in \mathcal{N}} a_{m, n} h(k-m L N / 2) e^{j \frac{2 \pi}{L N} n k} e^{j \varphi_{m n}}, \\
\forall k \in \mathcal{K} \triangleq\{1, \ldots, K\}
\end{array}
$$

where $a_{m, n}$ denotes the $m$-th real transmit symbol on subcarrier $n$ with $m \in \mathcal{M} \triangleq\{0,1, \ldots, 2 M-1\}$ and $n \in \mathcal{N}, h(k)$ is the prototype filter, and $\varphi_{m n}=\frac{(m+n) \pi}{2}-m n \pi$ is the phase factor. Moreover, (1) can be further expressed as the following matrix form:

$$
\mathbf{s}=\sum_{m=0}^{2 M-1} \mathbf{F}_{m} \mathbf{D} \mathbf{a}_{m}
$$

where $\quad \mathbf{F}_{m}=\left[\mathbf{0}_{\frac{m}{2}}^{T} L N \times N, \boldsymbol{\Phi}_{m}^{T} \mathbf{H}^{T}, \mathbf{0}_{\frac{2 M-m-1}{2} L N \times N}^{T}\right]^{T}$. The vector $\mathbf{s}=\left[s_{1}, s_{2}, \ldots, s_{K}\right]^{T}$ denotes the timedomain signal and $\mathbf{a}_{m}=\left[a_{m, 1}, a_{m, 2}, \ldots, a_{m, N}\right]^{T}$ denotes the real transmit symbols. The matrix $\mathbf{D}$ is a diagonal selection matrix with $D_{n, n}=1$ if $n \in \mathcal{N}$ and $D_{n, n}=0$ otherwise. Moreover, $\boldsymbol{\Phi}_{m}=$ $\operatorname{diag}\left[j^{m+0}(-1)^{0}, j^{m+1}(-1)^{m}, \cdots, j^{m+N-1}(-1)^{m(N-1)}\right]$ denotes the diagonal phase matrix, and $\mathbf{H} \in \mathbb{C}^{\beta L N \times N}$ denotes the pulse shaping matrix with elements $H_{p, q}=$ $h(p) e^{j \frac{2 \pi}{L N} p q}, p \in\{1, \ldots, \beta L N\}, q \in\{1, \ldots, N\}$.

Due to the overlap between adjacent symbols, we consider to minimize the PAPR of the whole FBMC frame. The framebased PAPR minimization problem is formulated as

$$
\begin{aligned}
\min _{\left\{\mathbf{a}_{m}, \mathbf{s}\right\}} & \frac{\|\mathbf{s}\|_{\infty}^{2}}{\frac{1}{K}\|\mathbf{s}\|_{2}^{2}} \\
\text { s.t. } & \mathbf{s}=\sum_{m=0}^{2 M-1} \mathbf{F}_{m} \mathbf{D} \mathbf{a}_{m}, \\
& \frac{\left\|\mathbf{D}\left(\mathbf{a}_{m}-\hat{\mathbf{a}}_{m}\right)\right\|_{2}^{2}}{\left\|\mathbf{D} \hat{\mathbf{a}}_{m}\right\|_{2}^{2}} \leq \gamma, \forall m, \\
& \frac{\left\|(\mathbf{I}-\mathbf{D}) \mathbf{a}_{m}\right\|_{2}^{2}}{\left\|\mathbf{D} \mathbf{a}_{m}\right\|_{2}^{2}} \leq \eta, \forall m,
\end{aligned}
$$

where $\hat{\mathbf{a}}_{m}$ denotes the original frequency symbols with no distortion, $\gamma$ is the pre-set threshold of the distortion-to-signal power ratio, and $\eta$ is the threshold of the dummy subcarrier power ratio. Constraint (3c) ensures that the distortion is below a threshold, while (3d) constrains the power allocated to the dummy subcarriers used for PAPR reduction. Problem (3) is challenging to handle due to the fractional form of the $\mathrm{OF}$ and owing to nonconvex constraints.

\section{Proposed PAPR Reduction Algorithm}

\section{A. Problem Transformation}

To optimize this fractional OF in (3), we introduce additional auxiliary variables $\{r, t, \tilde{t}\}$, where $r$ denotes the tight upper bound of $\|\mathbf{s}\|_{\infty}^{2}, \tilde{t}$ satisfies $\frac{1}{\tilde{t}} \geq \frac{r}{\|\mathbf{s}\|_{2}^{2}}$, and $t$ satisfies $t \geq \frac{K}{\tilde{t}}$. Then, by adopting the newly introduced auxiliary variables and inequalities as constraints, the original problem (3) can be equivalently converted to

$$
\begin{aligned}
\underset{\left\{\mathbf{a}_{m}, \mathbf{s}, r, t, \tilde{t}\right\}}{\min } & t \\
\text { s.t. } & r \tilde{t} \leq\|\mathbf{s}\|^{2}, t \tilde{t} \geq K,\left|s_{k}\right|^{2} \leq r, \forall k, \\
& (3 \mathrm{~b})-(3 \mathrm{~d}),
\end{aligned}
$$

where $s_{k}$ denotes the $k$-th element of $\mathbf{s}$. The original problem (3) is transformed into a more tractable form, i.e., (4). In particular, problem (4) is identical to problem (3) in the sense that the global optimal solutions for the variables $\left\{\mathbf{a}_{m}, \mathbf{s}\right\}$ are identical. It can be observed that problem (4) satisfies the framework of penalty-CCCP shown in [13].

\section{B. Proposed Penalty-CCCP based Algorithm}

In this subsection, we develop a penalty-CCCP based algorithm to solve problem (4).

1) Penalized Problem: In the penalty-CCCP framework, the equality constraints are incorporated into the $\mathrm{OF}$ as penalty terms [13]. The penalty-CCCP algorithm has a twin-loop structure. The penalty parameter is updated in the outer loop and the variables are updated in a block coordinate descent method in the inner loop. Therefore, by properly introducing auxiliary variables and equality constraints, we decompose the original problem into several subproblems, where each variable at most appears in one inequality constraint so that the subproblems can be solved by using the Lagrange multiplier method or the first-order optimality condition. Based on this guideline, we introduce the auxiliary variables $\left\{\tilde{\mathbf{s}}, \bar{r}_{k}, \bar{t}, \tilde{\mathbf{a}}_{m}, \overline{\mathbf{a}}_{m}\right\}$ that satisfy

$$
\tilde{\mathbf{s}}=\mathbf{s}, \bar{r}_{k}=r, \bar{t}=\tilde{t}, \tilde{\mathbf{a}}_{m}=\mathbf{a}_{m}, \overline{\mathbf{a}}_{m}=\mathbf{a}_{m}, \forall k, m
$$

Then, problem (4) can be equivalently transformed as

$$
\begin{array}{rl}
\min _{\mathcal{Z}} & t \\
\text { s.t. } & \left|s_{k}\right|^{2} \leq \bar{r}_{k}, \forall k, \\
& r \tilde{t} \leq\|\tilde{\mathbf{s}}\|^{2}, \\
& t \bar{t} \geq K, \\
& \left\|\mathbf{D}\left(\overline{\mathbf{a}}_{m}-\hat{\mathbf{a}}_{m}\right)\right\|_{2}^{2}-\gamma\left\|\mathbf{D} \hat{\mathbf{a}}_{m}\right\|_{2}^{2} \leq 0, \forall m, \\
& \left\|(\mathbf{I}-\mathbf{D}) \tilde{\mathbf{a}}_{m}\right\|_{2}^{2}-\eta\left\|\mathbf{D} \tilde{\mathbf{a}}_{m}\right\|_{2}^{2} \leq 0, \forall m, \\
& (3 \mathrm{~b}),(5),
\end{array}
$$

where $\mathcal{Z} \triangleq\left\{\mathbf{a}_{m}, \mathbf{s}, r, t, \tilde{t}, \tilde{\mathbf{s}}_{,}, \bar{r}_{k}, \bar{t}, \tilde{\mathbf{a}}_{m}, \overline{\mathbf{a}}_{m}\right\}$ denotes the set of variables. 
By incorporating the penalty terms corresponding to the equality constraints (3b) and (5) into the $\mathrm{OF}$, we finally obtain the following penalized problem

$$
\begin{aligned}
\min _{\mathcal{Z}} \mathcal{L}(\mathcal{Z}) \triangleq & +\rho\left(\left\|\mathbf{s}-\sum_{m=0}^{2 M-1} \mathbf{F}_{m} \mathbf{D} \mathbf{a}_{m}\right\|^{2}+\|\mathbf{s}-\tilde{\mathbf{s}}\|^{2}\right. \\
& +\sum_{m=0}^{2 M-1}\left\|\mathbf{a}_{m}-\tilde{\mathbf{a}}_{m}\right\|^{2}+\sum_{m=0}^{2 M-1}\left\|\mathbf{a}_{m}-\overline{\mathbf{a}}_{m}\right\|^{2} \\
& \left.+\sum_{k=1}^{K}\left|r-\bar{r}_{k}\right|^{2}+|\bar{t}-\tilde{t}|^{2}\right) \\
\text { s.t. } & (6 \mathrm{~b})-(6 \mathrm{f}) .
\end{aligned}
$$

Based on the introduction in [13], we observe that the outer loop updates the penalty parameter $\rho$ to reduce the equality constraint violation. While the inner loop solves the penalized problem (7) with fixed $\rho$ via CCCP [14]. The proposed penalty-CCCP based algorithm for problem (6) is summarized in Algorithm 1.

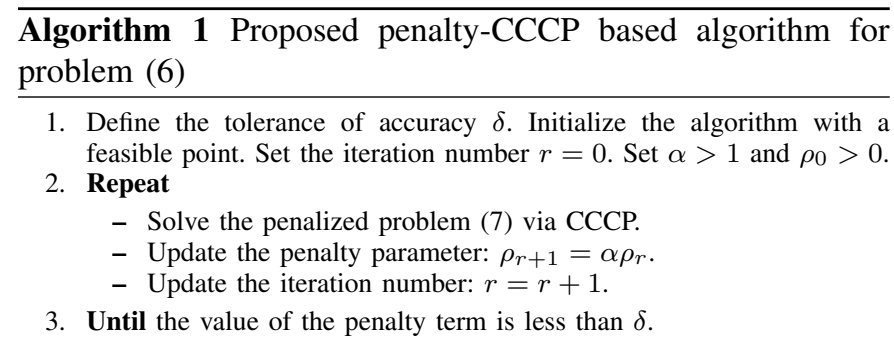

2) Proposed Algorithm for Solving Problem (7): Referring to the penalty-CCCP framework in [13], the core of the algorithm design is to develop an efficient algorithm to solve problem (7) with fixed $\rho$ for the inner loop.

Due to the nonconvex constraints (6c) and (6f), problem (7) is difficult to handle. However, we can successively approximate these nonconvex constraints as convex ones in each inner iteration according to the CCCP concept [14]. Let us take (6c) as an example. The nonconvex constraint (6c) can be rewritten as the following difference-of-convex (DC) form

$$
\frac{(r+\tilde{t})^{2}}{4}-\left(\frac{(r-\tilde{t})^{2}}{4}+\|\tilde{\mathbf{s}}\|_{2}^{2}\right) \leq 0 .
$$

Then, by applying the first-order Taylor expansion to linearize the subtracted convex term, we obtain the approximated convex constraint as follows

$$
\begin{array}{r}
\frac{(r+\tilde{t})^{2}}{4}-\left(\frac{\left(r^{i}-\tilde{t}^{i}\right)(r-\tilde{t})}{2}-\frac{\left(r^{i}-\tilde{t}^{i}\right)^{2}}{4}\right. \\
\left.+2 \Re\left\{\tilde{\mathbf{s}}^{i H} \tilde{\mathbf{s}}\right\}-\left\|\tilde{\mathbf{s}}^{i}\right\|^{2}\right) \leq 0,
\end{array}
$$

where $\left\{r^{i}, \tilde{t}^{i}, \tilde{\mathbf{s}}^{i}\right\}$ denotes the current point of $\{r, \tilde{t}, \tilde{\mathbf{s}}\}$ in the $i$-th inner iteration. Similarly, the nonconvex constraint (6f) can be approximated as the following convex one

$\left\|(\mathbf{I}-\mathbf{D}) \tilde{\mathbf{a}}_{m}\right\|_{2}^{2}-\eta\left(2 \Re\left\{\tilde{\mathbf{a}}_{m}^{i H} \mathbf{D}^{H} \mathbf{D} \tilde{\mathbf{a}}_{m}\right\}-\left\|\mathbf{D} \tilde{\mathbf{a}}_{m}^{i}\right\|_{2}^{2}\right) \leq 0, \forall m$.

Moreover, constraint (6d) can be rewritten as the following convex form

$$
\sqrt{K+\frac{(\bar{t}-t)^{2}}{4}}-\frac{\bar{t}+t}{2} \leq 0
$$

Hence, problem (7) can be approximated to the following convex problem in the $i$-th inner iteration:

$$
\begin{array}{ll}
\min _{\mathcal{Z}} & \mathcal{L}(\mathcal{Z}) \\
\text { s.t. } & (6 \mathrm{~b}),(6 \mathrm{e}),(9)-(11) .
\end{array}
$$

In the inner loop, we update the variables in a block coordinate descent method. In order to simplify the decomposed subproblems for each variable block, we consider the overlapping structure of FBMC and divide the variables into the following $2 \beta+2$ blocks:

- $\mathcal{Z}_{j}=\left\{\mathbf{a}_{2 \beta m^{\prime}+j-1}, m^{\prime}=0,1, \ldots,\left\lfloor\frac{2 M-j}{2 \beta}\right\rfloor\right\}, j \in \mathcal{J} \triangleq$ $\{1,2, \ldots, 2 \beta\}$,

- $\mathcal{Z}_{2 \beta+1}=\left\{\mathbf{s}, \bar{r}_{k}, t, \bar{t}\right\}$,

- $\mathcal{Z}_{2 \beta+2}=\left\{\tilde{\mathbf{s}}, r, \tilde{t}, \tilde{\mathbf{a}}_{m}, \overline{\mathbf{a}}_{m}\right\}$.

As a result, we express the $i$-th inner iteration as the $2 \beta+2$ steps shown below.

Step 1 to $2 \beta$ : update the variables in $\mathcal{Z}_{j}=$ $\left\{\overline{\mathbf{a}_{2 \beta m^{\prime}+j-1}, m^{\prime}}=0,1, \ldots,\left\lfloor\frac{2 M-j}{2 \beta}\right\rfloor\right\}$ with the other variables fixed in Step $\mathbf{j}$, where $j \in \mathcal{J}$. In Step $\mathbf{j}$, problem (12) can be decomposed into $\left\lfloor\frac{2 M-j}{2 \beta}\right\rfloor+1$ parallel subproblems, with the $m^{\prime}$-th subproblem expressed as

$$
\begin{aligned}
\min _{\mathbf{a}_{2 \beta m^{\prime}+j-1}} & \left\|\mathbf{s}-\sum_{m=2 \beta m^{\prime}+j-1}^{\min \left\{2 \beta m^{\prime}+j+2 \beta-2,2 M-1\right\}} \mathbf{F}_{m} \mathbf{D a} \mathbf{a}_{m}\right\|^{2} \\
& +\left\|\mathbf{a}_{2 \beta m^{\prime}+j-1}-\tilde{\mathbf{a}}_{2 \beta m^{\prime}+j-1}\right\|^{2} \\
& +\left\|\mathbf{a}_{2 \beta m^{\prime}+j-1}-\overline{\mathbf{a}}_{2 \beta m^{\prime}+j-1}\right\|^{2}
\end{aligned}
$$

By examining the first-order optimality condition with respect to $\mathbf{a}_{2 \beta m^{\prime}+j-1}$, we obtain the closed-form solution

$$
\begin{aligned}
& \mathbf{a}_{2 \beta m^{\prime}+j-1} \\
= & \left(\Re\left\{\mathbf{D}^{H} \mathbf{F}_{2 \beta m^{\prime}+j-1}^{H} \mathbf{F}_{2 \beta m^{\prime}+j-1} \mathbf{D}\right\}+2 \mathbf{I}\right)^{-1} \\
& \times\left(\tilde{\mathbf{a}}_{2 \beta m^{\prime}+j-1}+\overline{\mathbf{a}}_{2 \beta m^{\prime}+j-1}+\Re\left\{\mathbf{D}^{H} \mathbf{F}_{2 \beta m^{\prime}+j-1}^{H}\right.\right. \\
& \left.\left.\times\left(\mathbf{s}-\sum_{m=2 \beta m^{\prime}+j}^{\min \left\{2 \beta m^{\prime}+j+2 \beta-2,2 M-1\right\}} \mathbf{F}_{m} \mathbf{D} \mathbf{a}_{m}\right)\right\}\right) .
\end{aligned}
$$

Step $2 \boldsymbol{\beta}+1$ : update the variables in $\mathcal{Z}_{2 \beta+1}=\left\{\mathbf{s}, \bar{r}_{k}, t, \bar{t}\right\}$ with the other variables fixed. Problem (12) can be decomposed into two subproblems.

The first subproblem with respect to $\left\{\mathbf{s}, \bar{r}_{k}\right\}$ can be further divided into $K$ subproblems with the $k$-th one expressed as

$$
\begin{aligned}
\min _{s_{k}, \bar{r}_{k}} & \left|s_{k}-c_{k}\right|^{2}+\left|s_{k}-\tilde{s}_{k}\right|^{2}+\left|r-\bar{r}_{k}\right|^{2} \\
\text { s.t. } & \left|s_{k}\right|^{2} \leq \bar{r}_{k},
\end{aligned}
$$

where $\mathbf{c}=\sum_{m=0}^{2 M-1} \mathbf{F}_{m} \mathbf{D} \mathbf{a}_{m}$ is fixed in this step, and $c_{k}$ denotes the $k$-th element of $\mathbf{c}$. By attaching a Lagrange multiplier $\lambda_{k}$ to the constraint, we obtain the Lagrange function shown below

$$
\begin{array}{r}
\mathcal{L}\left(s_{k}, \bar{r}_{k}, \lambda_{k}\right) \triangleq\left|s_{k}-c_{k}\right|^{2}+\left|s_{k}-\tilde{s}_{k}\right|^{2}+\left|r-\bar{r}_{k}\right|^{2} \\
+\lambda_{k}\left(\left|s_{k}\right|^{2}-\bar{r}_{k}\right) .
\end{array}
$$

By applying the first-order optimality condition with respect to $s_{k}$ and $\bar{r}_{k}$, respectively, we obtain

$$
s_{k}\left(\lambda_{k}\right)=\frac{c_{k}+\tilde{s}_{k}}{2+\lambda_{k}}, \quad \bar{r}_{k}\left(\lambda_{k}\right)=r+\frac{\lambda_{k}}{2} .
$$


The value of the Lagrange multiplier $\lambda_{k}$ should be chosen to satisfy the complementarity slackness condition. Therefore, we first define the function corresponding to the constraint as

$$
\mathcal{C}\left(s_{k}, \bar{r}_{k}\right) \triangleq\left|s_{k}\right|^{2}-\bar{r}_{k}
$$

Then, we are able to obtain the value of $\lambda_{k}$ by examining the complementary slackness condition. That is, if $\mathcal{C}\left(s_{k}(0), \bar{r}_{k}(0)\right) \leq 0$ is satisfied, the optimal value of the multiplier is $\lambda_{k}=0$. Otherwise, we should search for a positive $\lambda_{k}$ that ensures

$$
\mathcal{C}\left(s_{k}\left(\lambda_{k}\right), \bar{r}_{k}\left(\lambda_{k}\right)\right)=\frac{\left(c_{k}+\tilde{s}_{k}\right)^{2}}{\left(2+\lambda_{k}\right)^{2}}-\frac{\lambda_{k}}{2}-r=0 .
$$

We are able to obtain the optimal $\lambda_{k}$ by applying the one dimensional bisection search to solve (19). Then, the optimal solution $\left\{s_{k}, \bar{r}_{k}\right\}$ is obtained by substituting the optimal $\lambda_{k}$ in (17).

Similarly, we use the Lagrange multiplier method to solve the second subproblem with respect to $\{t, \bar{t}\}$. The solution is

$$
t(\lambda)=\frac{\sqrt{K}(\lambda-2)}{\sqrt{\lambda-1}}+\frac{\lambda-1+2 \rho \tilde{t}}{2 \rho}, \quad \bar{t}(\lambda)=\frac{\lambda-1+2 \rho \tilde{t}}{2 \rho} .
$$

The value of $\lambda$ is obtained by examining the complementarity slackness condition corresponding to constraint (11).

Step $2 \boldsymbol{\beta}+2$ : update the variables in $\mathcal{Z}_{2 \beta+2}=$ $\left\{\tilde{\mathbf{s}}, r, \tilde{t}, \tilde{\mathbf{a}}_{m}, \overline{\mathbf{a}}_{m}\right\}$ with the other variables fixed. Similar to Step $2 \boldsymbol{\beta}+\mathbf{1}$, we decompose problem (12) into three subproblems with respect to $\{\tilde{\mathbf{s}}, r, \tilde{t}\},\left\{\tilde{\mathbf{a}}_{m}\right\}$, and $\left\{\overline{\mathbf{a}}_{m}\right\}$, respectively, and solve each of them by using the Lagrange multiplier method. is

The solution of the first subproblem with respect to $\{\tilde{\mathbf{s}}, r, \tilde{t}\}$

$$
\begin{gathered}
\tilde{\mathbf{s}}(\lambda)=\mathbf{s}+\lambda \tilde{\mathbf{s}}^{i} \\
r(\lambda)=\frac{8 \sum_{k=1}^{K} \bar{r}_{k}+2\left(r^{i}-\tilde{t}^{i}+\sum_{k=1}^{K} \bar{r}_{k}-\bar{t}\right) \lambda+\left(r^{i}-\tilde{t}^{i}\right) \lambda^{2}}{8 K+2(K+1) \lambda},
\end{gathered}
$$

and

$$
\tilde{t}(\lambda)=\frac{8 K \bar{t}+2\left(K\left(\tilde{t}^{i}-r^{i}\right)-\sum_{k=1}^{K} \bar{r}_{k}+\bar{t}\right) \lambda-\left(r^{i}-\tilde{t}^{i}\right) \lambda^{2}}{8 K+2(K+1) \lambda} .
$$

By examining the complementary slackness condition corresponding to constraint (9), the optimal value of $\lambda$ is obtained.

The second subproblem with respect to $\left\{\tilde{\mathbf{a}}_{m}\right\}$ can be further divided into $M$ subproblems. The solution of the $m$-th subproblem is

$$
\tilde{\mathbf{a}}_{m}\left(\lambda_{m}\right)=\left(\mathbf{I}+\lambda_{m}(\mathbf{I}-\mathbf{D})\right)^{-1}\left(\mathbf{a}_{m}+\eta \lambda_{m} \mathbf{D} \tilde{\mathbf{a}}_{m}^{i}\right) .
$$

The value of $\lambda_{m}$ is obtained by examining the complementary slackness condition corresponding to constraint (10). Note that the solution of $\left\{\tilde{\mathbf{a}}_{m}\right\}$ contains matrix and vector operations, which may lead to high complexity in the bisection search of $\lambda_{m}$. Hence, we convert the function corresponding to constraint (10) to an equivalent form that only contains scalar operations of $\lambda_{m}$ as shown in (25), where $d_{p}=[\mathbf{D}]_{p, p}, u_{1, p}=\left[\mathbf{a}_{m} \mathbf{a}_{m}^{H}\right]_{p, p}, u_{2, p}=\left[2 \eta \Re\left\{\mathbf{D} \tilde{\mathbf{a}}_{m}^{i} \mathbf{a}_{m}^{H}\right\}\right]_{p, p}$, $u_{3, p}=\left[\eta^{2} \mathbf{D} \tilde{\mathbf{a}}_{m}^{i} \tilde{\mathbf{a}}_{m}^{i H} \mathbf{D}^{H}\right]_{p, p}, \tilde{u}_{1, p}=\left[\Re\left\{\mathbf{a}_{m} \tilde{\mathbf{a}}_{m}^{i H}\right\}\right]_{p, p}, \tilde{u}_{2, p}=$ $\left[\eta \mathbf{D} \tilde{\mathbf{a}}_{m}^{i} \tilde{\mathbf{a}}_{m}^{i H}\right]_{p, p}$, and $\bar{u}=\eta\left\|\mathbf{D} \tilde{\mathbf{a}}_{m}^{i}\right\|_{2}^{2}$. If $\mathcal{C}\left(\tilde{\mathbf{a}}_{m}(0)\right) \leq 0$ is satisfied, the optimal value of the multiplier is $\lambda_{m}=0$. Otherwise, we apply the one dimensional bisection search to solve $\mathcal{C}\left(\tilde{\mathbf{a}}_{m}\left(\lambda_{m}\right)\right)=0$ and obtain a positive $\lambda_{m}$. By substituting the optimal $\lambda_{m}$ in (24), the optimal solution $\tilde{\mathbf{a}}_{m}$ is obtained.

The third subproblem with respect to $\left\{\overline{\mathbf{a}}_{m}\right\}$ can be further divided into $M$ subproblems. The solution of the $m$-th subproblem is

$$
\overline{\mathbf{a}}_{m}\left(\lambda_{m}\right)=\left(\mathbf{I}+\lambda_{m} \mathbf{D}\right)^{-1}\left(\mathbf{a}_{m}+\lambda_{m} \mathbf{D} \hat{\mathbf{a}}_{m}\right) .
$$

Again, the value of $\lambda_{m}$ is obtained by examining the complementary slackness condition corresponding to constraint (6e).

\section{Convergence and Complexity}

As demonstrated in [13], the proposed penalty-CCCP based algorithm converges to a stationary solution of problem (6). Note that (3) and (6) are equivalent in the sense of the solution $\left\{\mathbf{a}_{m}, \mathbf{s}\right\}$. Therefore, the obtained solution is also a stationary point of the original problem (3).

The complexity is measured by the number of complex multiplications. Let us focus on the updating of $\left\{\mathbf{a}_{m}\right\}$ in Step 1 to $2 \boldsymbol{\beta}$ and $\left\{\tilde{\mathbf{a}}_{m}\right\}$ in Step $2 \boldsymbol{\beta}+\mathbf{2}$, respectively. The variables $\left\{\mathbf{a}_{m}\right\}$ are updated through (14). Note that $\Re\left\{\mathbf{D}^{H} \mathbf{F}_{2 \beta m^{\prime}+j-1}^{H} \mathbf{F}_{2 \beta m^{\prime}+j-1} \mathbf{D}\right\}$ is a constant diagonal matrix, which can be precalculated, since the matrices are invariant and FBMC achieves real-domain orthogonal. Moreover, the operation $\Re\left\{\mathbf{D}^{H} \mathbf{F}_{2 \beta m^{\prime}+j-1}^{H} \mathbf{s}\right\}$ is equivalent to demodulating the signal $\mathbf{s}$, which requires a complexity of $\mathcal{O}\left(\beta L N+L N \log _{2}(L N)\right)$ for each symbol block when employing the PPN structure [1]. Similarly, the complexity of calculating $\Re\left\{\mathbf{D}^{H} \mathbf{F}_{2 \beta m^{\prime}+j-1}^{H} \sum_{m=2 \beta m^{\prime}+j}^{\min \left\{2 \beta m^{\prime}+j+2 \beta-2,2 M-1\right\}} \mathbf{F}_{m} \mathbf{D} \mathbf{a}_{m}\right\}$ is also $\mathcal{O}\left(\beta L N+L N \log _{2}(L N)\right)$. Therefore, the overall computational complexity of updating $\left\{\mathbf{a}_{m}\right\}, \forall m$, is given by $\mathcal{O}\left(M \beta L N+M L N \log _{2}(L N)\right)$ after excluding the precalculations corresponding to the invariant matrices. For the subproblems with respect to $\left\{\tilde{\mathbf{a}}_{m}\right\}$, we should first solve $\mathcal{C}\left(\tilde{\mathbf{a}}_{m}\left(\lambda_{m}\right)\right)=0$ to find the optimal $\lambda_{m}$ and then compute $\left\{\tilde{\mathbf{a}}_{m}\left(\lambda_{m}\right)\right\}$ through (24). The complexity of solving $\mathcal{C}\left(\tilde{\mathbf{a}}_{m}\left(\lambda_{m}\right)\right)=0$ is dominated by computing the coefficients in (25) and the one dimensional bisection search. Since $\mathbf{D}$ is a diagonal selecting matrix, computing the coefficients in (25) requires a complexity of $\mathcal{O}(N)$. Moreover, the complexity of the one dimensional bisection search is given by $\mathcal{O}\left(\log _{2}\left(\frac{\theta_{0}}{\theta}\right)\right)$, where $\theta_{0}$ is the initial interval size and $\theta$ is the tolerance. Hence, the complexity of solving $\mathcal{C}\left(\tilde{\mathbf{a}}_{m}\left(\lambda_{m}\right)\right)=0$ is given by $\mathcal{O}\left(\log _{2}\left(\frac{\theta_{0}}{\theta}\right)+N\right)$. After obtaining the optimal $\lambda_{m}$, the complexity of computing $\left\{\tilde{\mathbf{a}}_{m}\left(\lambda_{m}\right)\right\}$ through (24) is $\mathcal{O}(N)$. Therefore, the overall complexity of updating $\left\{\tilde{\mathbf{a}}_{m}\right\}, \forall m$ is given by $\mathcal{O}\left(M \log _{2}\left(\frac{\theta_{0}}{\theta}\right)+M N\right)$.

By following the similar procedures of analyzing the complexity of updating $\left\{\mathbf{a}_{m}\right\}$ and $\left\{\tilde{\mathbf{a}}_{m}\right\}$, the complexity of each subproblem in the inner loops can be obtained. By retaining dominant terms, the overall complexity of each inner iteration for the proposed algorithm 


$$
\begin{aligned}
\mathcal{C}\left(\tilde{\mathbf{a}}_{m}\left(\lambda_{m}\right)\right) \triangleq & \left\|(\mathbf{I}-\mathbf{D}) \tilde{\mathbf{a}}_{m}\right\|_{2}^{2}-\eta\left(2 \Re\left\{\tilde{\mathbf{a}}_{m}^{i H} \mathbf{D}^{H} \mathbf{D} \tilde{\mathbf{a}}_{m}\right\}-\left\|\mathbf{D} \tilde{\mathbf{a}}_{m}^{i}\right\|_{2}^{2}\right), \\
= & \operatorname{Tr}\left\{\left(\mathbf{a}_{m}+\eta \lambda_{m} \mathbf{D} \tilde{\mathbf{a}}_{m}^{i}\right)^{H}\left(\mathbf{I}+\lambda_{m}(\mathbf{I}-\mathbf{D})\right)^{-H}(\mathbf{I}-\mathbf{D})\left(\mathbf{I}+\lambda_{m}(\mathbf{I}-\mathbf{D})\right)^{-1}\left(\mathbf{a}_{m}+\eta \lambda_{m} \mathbf{D} \tilde{\mathbf{a}}_{m}^{i}\right)\right\} \\
& -\operatorname{Tr}\left\{2 \eta \Re\left\{\tilde{\mathbf{a}}_{m}^{i H} \mathbf{D}\left(\mathbf{I}+\lambda_{m}(\mathbf{I}-\mathbf{D})\right)^{-1}\left(\mathbf{a}_{m}+\eta \lambda_{m} \mathbf{D} \tilde{\mathbf{a}}_{m}^{i}\right)\right\}\right\}+\eta\left\|\mathbf{D} \tilde{\mathbf{a}}_{m}^{i}\right\|_{2}^{2} \\
= & \sum_{p=1}^{N} \frac{\left(1-d_{p}\right)\left(u_{1, p}+u_{2, p} \lambda_{m}+u_{3, p} \lambda_{m}^{2}\right)}{\left(1+\left(1-d_{p}\right) \lambda_{m}\right)^{2}}-\sum_{p=1}^{N} \frac{2 \eta d_{p}\left(\tilde{u}_{1, p}+\tilde{u}_{2, p} \lambda_{m}\right)}{\left(1+\left(1-d_{p}\right) \lambda_{m}\right)}+\bar{u}
\end{aligned}
$$

is $\mathcal{O}\left(\beta L M N+L M N \log _{2}(L N)+(M+\beta) L N \log _{2}\left(\frac{\theta_{0}}{\theta}\right)\right)$. Note that the complexity of bisection search, i.e., $\log _{2}\left(\frac{\theta_{0}}{\theta}\right)$, is generally small and negligible if $M$ and $N$ are large enough. Hence, the overall computational complexity of the proposed algorithm is roughly expressed as $\mathcal{O}\left(I_{1} I_{2}\left(\beta L M N+L M N \log _{2}(L N)\right)\right)$, where $I_{1}$ and $I_{2}$ denote the maximum inner and outer iteration numbers, respectively.

If problem (12) in each inner iteration is solved via the traditional interior-point method, the overall complexity is roughly $\mathcal{O}\left(I_{1} I_{2}(L M N)^{3.5}\right)$ [15]. The complexity order of our proposed algorithm is much lower than the traditional interior-point method since all the variables are updated via the closed-form solutions or the Lagrange multiplier method.

\section{Simulation Results}

The FBMC system is assumed to have $N=64$ subcarriers, where 52 of them are data subcarriers and the rest 12 are dummy subcarriers. There are $M=10$ FBMC symbol blocks in one frame. Here we use the same prototype filter as in [1] and set the overlapping factor as $\beta=4$. Moreover, the oversampling rate is $L=4$. For the penalty-CCCP based algorithm, we set the initial penalty parameter as $\rho_{0}=10^{-3}$ and the increasing parameter $\alpha=2$. The maximum number of inner iterations is $N_{\max }=100$.

Fig. 1 illustrates the convergence performance of the proposed algorithm. The thresholds of distortion on data subcarriers and power on dummy subcarrier are chosen as $\gamma=0.01$ and $\eta=0.09$, respectively. From the figure, we observe that the PAPR value decreases with the increasing of outer iterations and the algorithm converges within 20 iterations. Moreover, the penalty value decreases below $10^{-8}$ within 30 iterations, which demonstrates that the proposed penaltyCCCP based algorithm effectively handles the complicated constraints of the optimization problem.

Fig. 2 shows the PAPR complementary cumulative distribution functions (CCDFs) and BER performance for different schemes, including the following algorithms for comparison:

- Original: the original FBMC signal with no distortion.

- Proposed: the proposed penalty-CCCP based algorithm.

- TR: the tone reservation method, which iteratively clips the signal and constructs peak cancelling signal on dummy subcarriers [11].

- ICF: the iterative clipping and filtering method, which iteratively clips the signal and employs the demodulated result as the transmit symbols.
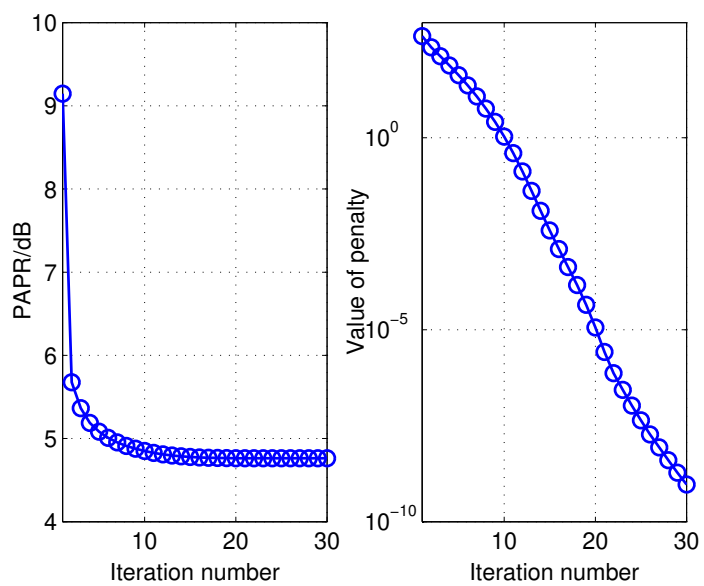

Fig. 1: The convergence performance of the proposed penalty-CCCP based algorithm.

The algorithms compared here reduce the PAPR by introducing signal distortion. Hence, the PAPR of FBMC signals can be better suppressed if larger distortion is permitted. However, the BER performance degrades at the same time. It is worth noting that if the distortion is well designed, a better tradeoff between the PAPR and BER performance could be achieved. The clipping threshold is denoted as $A$. When CCDF $=10^{-3}$, our proposed algorithm outperforms TR even if TR achieves its best performance with $A=1.8$. Moreover, our proposed algorithm also outperforms the ICF method when $A=1.8$. When the clipping threshold is $A=1.8$ and $\mathrm{SNR}=10 \mathrm{~dB}$, the BER performance degradation of our proposed algorithm is small compared to the TR method. Moreover, our algorithm outperforms the ICF method.

From the simulations above, we observe that our proposed method outperforms ICF both in PAPR performance and BER performance. When compared with TR, our algorithm significantly reduces the PAPR with slightly degraded BER performance.

\section{CONCLUSION}

In this paper, we have investigated the reduction of PAPR for FBMC systems. In particular, we have minimized the PAPR of a frame of FBMC signals with the constraint of the distortion on data subcarriers and the power constraint on dummy subcarriers. In order to handle the nonconvex problem, we have transformed the original problem into a more tractable form and then proposed a penalty-CCCP based algorithm. Simulation results show that the proposed algorithm effectively 

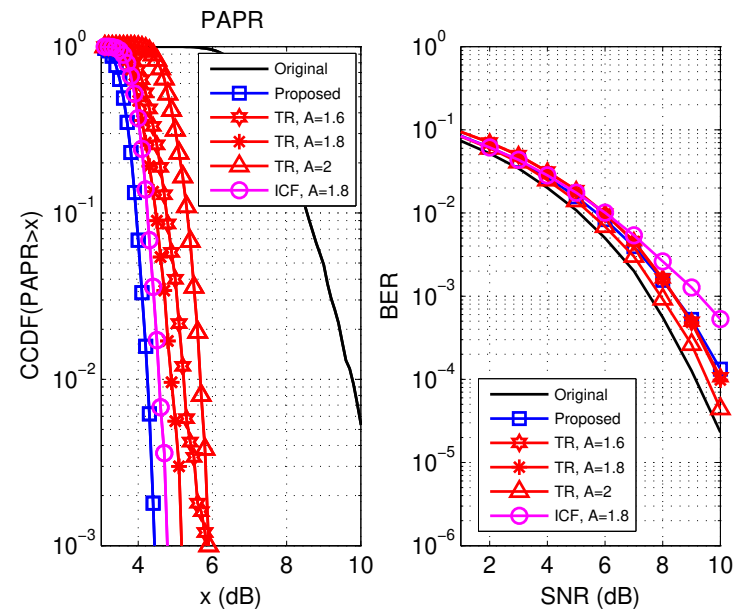

Fig. 2: PAPR reduction performance and BER performance for different schemes.

reduces the PAPR of FBMC signals with slightly reduced BER performance.

\section{REFERENCES}

[1] H. Saeedi-Sourck, Y. Wu, J. W. M. Bergmans, S. Sadri, and B. FarhangBoroujeny, "Complexity and performance comparison of filter bank multicarrier and OFDM in uplink of multicarrier multiple access networks," IEEE Trans. Signal Process., vol. 59, no. 4, pp. 1907-1912, Apr. 2011.

[2] Y. Cai, Z. Qin, F. Cui, G. Y. Li, and J. A. McCann, "Modulation and multiple access for 5G networks," IEEE Commun. Surv. Tut., vol. 20, no. 1, pp. 629-646, first quarter 2018.

[3] L. Hanzo, M Mnster, B Choi, and T Keller, OFDM and MC-CDMA for broadband multi-user communications, WLANs and broadcasting. John Wiley \& Sons, 2005.

[4] Y. (G.) Li and G. Stuber, OFDM for Wireless Communications (edited), Springer, Inc., Boston, MA, Jan. 2006.

[5] B. Li, W. Xu, H. Zhang, C. Zhao, and L. Hanzo, "PAPR reduction for hybrid ACO-OFDM aided IM/DD optical wireless vehicular communications," IEEE Trans. Veh. Technol., vol. 66, no. 10, pp. 9561-9566, Oct. 2017.

[6] Y. Wang, Y. Wang, and Q. Shi, "Optimized signal distortion for PAPR reduction of OFDM signals with IFFT/FFT complexity Via ADMM approaches," IEEE Trans. Signal Process., vol. 67, no. 2, pp. 399-414, Jan. 2019.

[7] D. Qu, S. Lu, and T. Jiang, "Multi-block joint optimization for the peakto-average power ratio reduction of FBMC-OQAM signals," IEEE Trans. Signal Process., vol. 61, no. 7, pp. 1605-1613, Apr. 2013.

[8] Z. He, L. Zhou, Y. Chen, and X. Ling, "Low-complexity PTS scheme for PAPR reduction in FBMC-OQAM systems," IEEE Commun. Lett., vol. 22, no. 11, pp. 2322-2325, Nov. 2018.

[9] R. Nissel and M. Rupp, "Pruned DFT-spread FBMC: Low PAPR, low latency, high spectral efficiency," IEEE Trans. Commun., vol. 66, no. 10, pp. 4811-4825, Oct. 2018.

[10] D. Na and K. Choi, "Low PAPR FBMC," IEEE Trans. Wireless Commun., vol. 17, no. 1, pp. 182-C193, Jan. 2018.

[11] S. Ren, H. Deng, X. Qian, and Y. Liu, "Sparse PTS scheme based on TR schemes for PAPR reduction in FBMC-OQAM systems," IET Commun., vol. 12, no. 14, pp. 1722-1727, Aug. 2018.

[12] J. Zhao, S. Ni, and Y. Gong, "Peak-to-average power ratio reduction of FBMC/OQAM signal using a joint optimization scheme," IEEE Access, vol. 5, pp. 15810-15819, May 2017.

[13] Y. Cai, Q. Shi, B. Champagne, and Y. (G.) Li, "Joint transceiver design for secure downlink communications over an amplify-and-forward MIMO relay," IEEE Trans. Commun., vol. 65, no. 9, pp. 3691-3704, Sep. 2017.

[14] A. L. Yuille and A. Rangarajan, "The concave-convex procedure," Neural Comput., vol. 15, no. 4, pp. 915-936, 2003.

[15] K.-Y. Wang, A. Man-Cho So, T.-H. Chang, W.-K. Ma, and C.-Y. Chi, "Outage constrained robust transmit optimization for multiuser MISO downlinks: Tractable approximations by conic optimization," IEEE Trans. Signal Process., vol. 62, no. 21, pp. 5690-5705, Nov. 2014. 